\title{
An Improved Ant-Based Algorithm for Minimum Degree Spanning Tree Problems
}

\author{
Md. Niaz Imtiaz ${ }^{1}$, Md.Akkas Ali ${ }^{2}$ \\ ${ }^{1,2}$ Lecturer, Dept. of CSE, University of Information Technology \& Sciences (UITS), Baridhara, Dhaka-1212, \\ Bangladesh
}

\begin{abstract}
A spanning tree of à connected graph is à sub graph, with least number of edges that still spans. The problem of finding degree constraint spanning tree is known to be NP-hard. In this paper we discuss an Ant-Based algorithm for finding minimum degree spanning trees and give improvement of the algorithm. We also show comparisons among the three algorithms and find the best improved Ant-Based algorithm. Extensive experimental results show that our improved algorithm performs very well against other algorithms on a set of 50 problem instances.
\end{abstract}

Keywords - Ant algorithm, Graph algorithms, Heuristic methods, minimum degree spanning tree,

\section{INTRODUCTION}

This paper describes Ant-Based algorithms for minimum degree spanning tree (AB-MDST) of unweighted connected graph. This is an interesting, real-world problem that seems well suited to an ant algorithm approach. The AB-MDST problem entails finding a spanning tree such that the maximum degree of a vertex in the tree becomes minimum [2]. This concept is useful in the design of telecommunication networks, design of networks for computer communication, design of integrated circuits, energy networks, transportation, logistics, and sewage networks [3]. For instance, switches in an actual communication network will have limited number of connections available. Transportation systems must place a limit on the number of roads meeting in one place.

The problem of finding degree constraint spanning tree is NP-hard [9]. Therefore, heuristics are often used to find good solutions in a reasonable amount of time [10]. We have used one type of heuristic called Ant Colony Optimization (ACO) [10]. Here, artificial ants move based on local information and pheromone levels. Our algorithm uses cumulative pheromone levels to determine candidate set of edges from which minimum degree spanning trees are built [7]. In this paper, we compare 3 algorithms - AB-MDST without local search and without degree constraint, AB-MDST with local search but without degree constraint and the last one ABMDST with local search and with degree constraint. Extensive experimental results show that AB-MDST with local search and with degree constraint performs very well against other algorithms.

The rest of the paper is organized as follow. In section 2 Our Ant-Based algorithm and two improved versions of that algorithm are described. Section 3 compares the performances of the three algorithms. The conclusion is given in section 4 .

\subsection{Initialization}

II. Methodology

Initially one ant is assigned to each vertex of the graph. In the next step, pheromone is assigned to each edge using the formula $P[i][j]=(M-d[j])+(M-m) / 3$, where $P[i][j]$ is pheromone level of edge $(i, j), d[j]$ is degree of $\mathrm{j}$ vertex, $\mathrm{M}$ is maximum degree of the input Graph and $\mathrm{m}$ is minimum degree of the input Graph [6]. Note that if a vertex has smaller degree, the edges connected to that vertex have higher pheromone levels.

\subsection{Exploration}

In each step each ant moves along one of the adjacent edge of its present vertex and after moving, the pheromone level of that edge is enhanced using the formula $P[i][j]=(M-d[j])+(M-m) / 3$. This event occurs until all the ants visit all the vertices [10]. In the tree construction section, a set $\mathrm{C}$ of candidate edges based on pheromone levels is identified. From $\mathrm{C}$ a spanning tree $\mathrm{T}$ is constructed. After constructing spanning tree the maximum degree cost $(T)$ of the spanning tree $T$ is calculated. Next, cost $(T)$ is compared with cost $(B)$, where cost (B) is the previous best tree (whose maximum degree is minimum) [5]. If cost (T) is smaller than cost (B), $\mathrm{T}$ is the present best tree and cost $(\mathrm{T})$ is assigned to cost (B), because we always try to find the best tree whose degree is minimum. The pheromone level for edges in the best tree B is then enhanced. This entire event is repeated until the stopping criteria met. 
2.3 Ant Movement

Let, an ant $\alpha$ is at vertex $i$. In case of AB-MDST without local search, an edge (i,j) is selected randomly, where $j$ is an adjacent vertex of $i$. In case of AB-MDST with local search, all the adjacent edges of vertex $i$ are considered, then the edge with highest pheromone level is selected. Note that, among all the adjacent edges the edge $(\mathrm{i}, \mathrm{j})$ has highest pheromone level if the degree of vertex $\mathrm{j}$ is minimum [4]. For this reason in case of AB-MDST with local search, better result is found than in the case of AB-MDST without local search. After an edge (i,j) is selected, ant $\alpha$ moves from vertex $i$ to vertex $j$ and the pheromone level of the edge $(i, j)$ is enhanced and vertex $\mathrm{j}$ is then marked as visited .

\subsection{Tree Construction}

After the ants have completed their movements and the pheromone levels of all the edges are updated, we are ready to identify the edges from which to construct a spanning tree. To identify a set of candidate edges, we first sort the edges in the graph in the order of descending pheromone level. The top candidate edges from the sorted list are selected to form candidate set C. During constructing tree, edges are taken one by one from C maintaining the order [9]. Let $(i, j)$ be next candidate edge and $i$ and $j$ are not connected in $T$. In case of ABMDST without degree constraint, the edge (i,j) is removed from $\mathrm{C}$ and added to tree $\mathrm{T}$ if this would create no loop. In case of AB-MDST with degree constraint, while adding $(\mathrm{i}, \mathrm{j})$ edge to tree $\mathrm{T}$ another checking is needed. If after adding ( $\mathrm{i}, \mathrm{j})$, degree of $\mathrm{i}$ or degree of $\mathrm{j}$ exceeds given parameter $\mathrm{k}$ and number of skipped edges(skippedEdge) $\mathrm{C}$ is smaller than $\mathrm{E}-\mathrm{V}$, the edge, $(\mathrm{i}, \mathrm{j})$ is skipped and added to skippedEdge rather than adding it to tree $\mathrm{T}$ and each time $\mathrm{C}$ is increased by one [8]. This event continues until the entire tree is constructed.

\subsection{Stopping Criteria}

The algorithm stops if one of the following two conditions is satisfied: 1. there is no improvement found in 1,000 consecutive cycles, or 2 . It has run for 5,000 cycles. When the algorithm stops, the current best tree is returned.

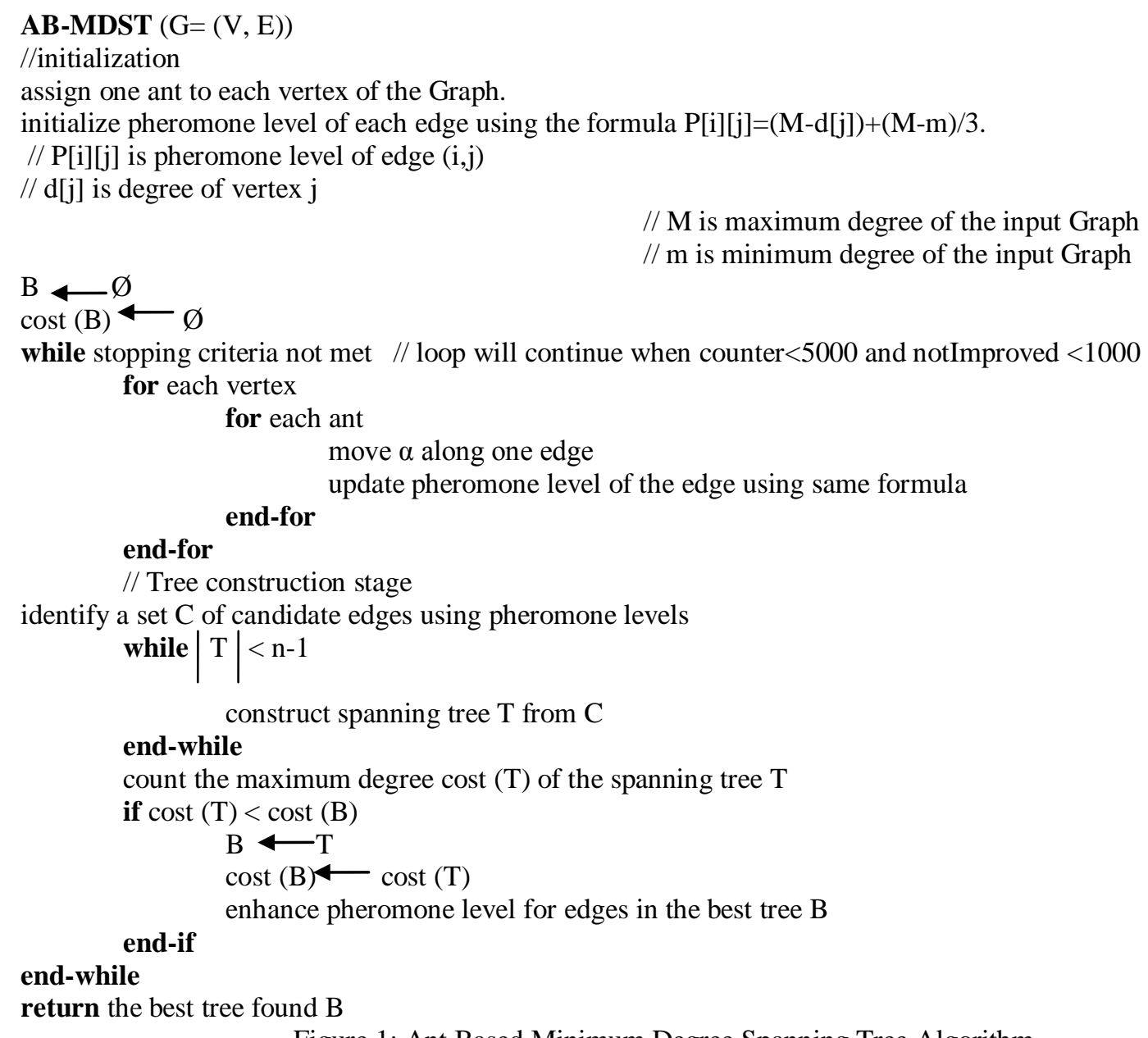

Figure 1: Ant Based Minimum Degree Spanning Tree Algorithm

Move $(\alpha, \mathrm{i}) \quad / /$ ant $\alpha$ is at vertex $\mathrm{i}$ 
select an adjacent edge $(\mathrm{i}, \mathrm{j}) / / \mathrm{j}$ is an adjacent vertex of $\mathrm{i}$ vertex

if vertex $\mathrm{j}$ is unvisited update pheromone level of edge $(i, j)$ move $\alpha$ from vertex $i$ to vertex $\mathrm{j}$ mark $\mathrm{j}$ visited break

else

end-if

$\alpha$ remains at vertex $\mathrm{i}$

Figure 2: One Step in the ant movement algorithm (AB-MDST without local search)

ConstructTree $(\mathrm{G}=(\mathrm{V}, \mathrm{E}))$

sort all the edges by pheromone level in descending order

$\mathrm{C} \longleftarrow$ top candidate edges ( highest pheromone levels )

$\mathrm{T}$

while $|\mathrm{T}|<\mathrm{n}-1$

let $(\mathrm{i}, \mathrm{j})$ be next candidate edge

if $i$ and $j$ not connected in $T$

if adding $(\mathrm{i}, \mathrm{j})$ to $\mathrm{T}$ would create no loop remove $(i, j)$ from $C$

end-if add $(i, j)$ edge to tree $T$

end-if

end-while

return $\mathrm{T}$

Figure 3: Tree construction (AB-MDST without degree constraint)

Move $(\alpha, \mathrm{i}) / /$ ant $\alpha$ is at vertex $\mathrm{i}$

find all the adjacent edges of verrex $i$

count the number of adjacent edges

if count $=0$

ant $\alpha$ remains at vertex $i$

else if count $=1$

select the adjacent edge $(\mathrm{i}, \mathrm{j})$

else

find the adjacent edge $(i, j)$ whose pheromone level is maximum

select the adjacent edge $(\mathrm{i}, \mathrm{j})$

end-if

if vertex $\mathrm{j}$ is unvisited

update pheromone level of edge $(i, j)$

move $\alpha$ from vertex $i$ to vertex $j$

mark $\mathrm{j}$ visited

break

else

$\alpha$ remains at vertex $\mathrm{i}$

end-if

Figure 4: One step in the ant movement algorithm (AB-MDST with local search)

ConstructTree $(\mathrm{G}=(\mathrm{V}, \mathrm{E}), \mathrm{k}) \quad / / \mathrm{k}$ is degree constraint

sort all the edges by pheromone level in descending order

$\mathrm{C} \longleftarrow$ top candidate edges ( highest pheromone levels )

$\mathrm{T}$

while $|\mathrm{T}|<\mathrm{n}-1$

let $(\mathrm{i}, \mathrm{j})$ be next candidate edge

if $\mathrm{i}$ and $\mathrm{j}$ not connected in $\mathrm{T}$

if degree [i] $>\mathrm{k}$ or degree $[\mathrm{j}]>\mathrm{k}$

and $\mathrm{C}<\mathrm{E}-\mathrm{V} \quad / / \mathrm{C}$ is number of skipped edges

add $(\mathrm{i}, \mathrm{j})$ to skippededge 


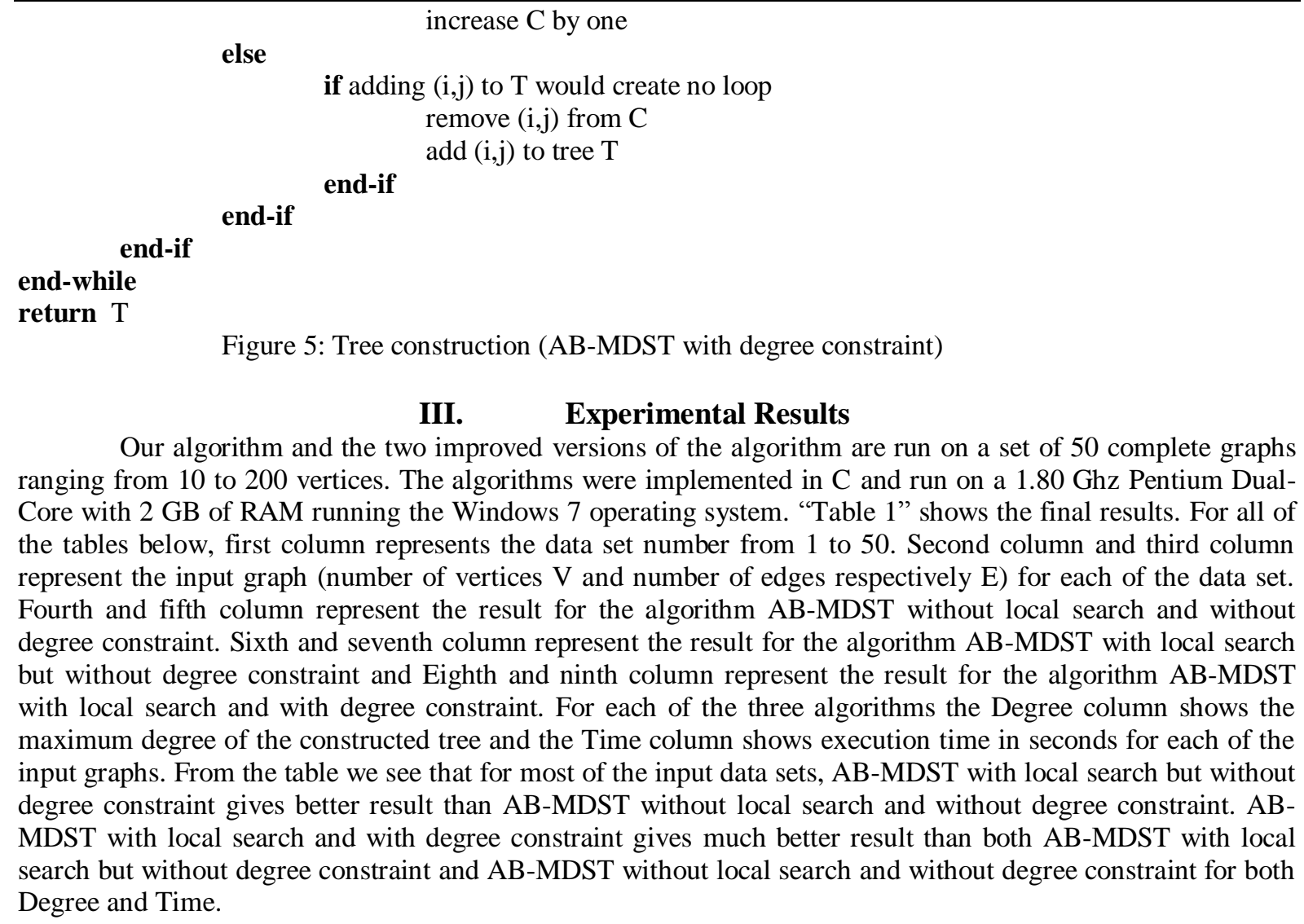

Input Graph

$\begin{array}{lll}\text { Dataset } & \text { V } & \text { E } \\ \text { Data1 } & 10 & 21 \\ \text { Data2 } & 10 & 22 \\ \text { Data3 } & 10 & 24 \\ \text { Data4 } & 10 & 25 \\ \text { Data5 } & 10 & 26 \\ \text { Data6 } & 10 & 27 \\ \text { Data7 } & 10 & 34 \\ \text { Data8 } & 25 & 69 \\ \text { Data9 } & 25 & 70 \\ \text { Data10 } & 28 & 75 \\ \text { Data11 } & 25 & 72 \\ \text { Data12 } & 25 & 90 \\ \text { Data13 } & 25 & 71 \\ \text { Data14 } & 43 & 63 \\ \text { Data15 } & 45 & 85 \\ \text { Data16 } & 50 & 123 \\ \text { Data17 } & 50 & 145 \\ \text { Data18 } & 60 & 166 \\ \text { Data19 } & 50 & 157 \\ \text { Data20 } & 50 & 183 \\ \text { Data21 } & 50 & 491 \\ \text { Data22 } & 50 & 582 \\ \text { Data23 } & 50 & 171 \\ \text { Data24 } & 75 & 196 \\ \text { Data25 } & 75 & 215 \\ \text { Data26 } & 75 & 256 \\ \text { Data27 } & 75 & 202\end{array}$

Table 1: Experimental Results

Output

\begin{tabular}{llllll}
\multicolumn{5}{c}{ Algorithm 1 Algorithm 2 } & \multicolumn{3}{c}{ Algorithm 3 } \\
Degree & Time & Degree & Time & Degree & Time \\
3 & 0 & 3 & 0 & 2 & 0 \\
3 & 0.016 & 3 & 0.016 & 2 & 0 \\
2 & 0 & 2 & 0 & 2 & 0 \\
2 & 0 & 3 & 0.02 & 2 & 0 \\
3 & 0 & 3 & 0 & 2 & 0 \\
3 & 0.016 & 3 & 0 & 2 & 0 \\
2 & 0 & 3 & 0 & 2 & 0 \\
3 & 0.078 & 3 & 0.047 & 2 & 0 \\
3 & 0.078 & 3 & 0.062 & 2 & 0 \\
4 & 0.094 & 3 & 0.078 & 2 & 0 \\
3 & 0.078 & 3 & 0.063 & 2 & 0.094 \\
3 & 0.078 & 3 & 0.078 & 2 & 0.01 \\
3 & 0.094 & 3 & 0.078 & 2 & 0 \\
4 & 0.25 & 5 & 0.203 & 3 & 0.203 \\
3 & 0.297 & 2 & 0 & 3 & 0.171 \\
5 & 0.486 & 3 & 0.359 & 3 & 0.235 \\
3 & 0.453 & 4 & 0.375 & 3 & 0.234 \\
4 & 0.719 & 2 & 0 & 3 & 0.359 \\
4 & 0.437 & 4 & 0.321 & 3 & 0.282 \\
4 & 0.453 & 4 & 0.328 & 3 & 0.265 \\
4 & 0.625 & 5 & 0.485 & 3 & 0.359 \\
3 & 0.593 & 3 & 0.469 & 3 & 0.344 \\
4 & 0.437 & 3 & 0.359 & 3 & 0.235 \\
4 & 1.359 & 4 & 0.984 & 3 & 0.703 \\
5 & 1.531 & 5 & 1.547 & 3 & 0.781 \\
4 & 1.36 & 4 & 1.265 & 3 & 0.703 \\
4 & 1.375 & 4 & 0.906 & 3 & 0.672
\end{tabular}


An Improved Ant-Based Algorithm for Minimum Degree Spanning Tree Problems

\begin{tabular}{|c|c|c|c|c|c|c|c|c|}
\hline Data28 & 75 & 266 & 5 & 1.547 & 5 & 1.094 & 3 & 0.797 \\
\hline Data29 & 100 & 297 & 5 & 2.922 & 5 & 2.828 & 3 & 1.532 \\
\hline Data30 & 100 & 324 & 4 & 3.125 & 5 & 2.578 & 3 & 1.578 \\
\hline Data31 & 100 & 334 & 4 & 4.563 & 5 & 3.734 & 3 & 2.047 \\
\hline Data32 & 100 & 314 & 3 & 3.062 & 6 & 4.969 & 3 & 1.547 \\
\hline Data33 & 100 & 394 & 4 & 3.862 & 4 & 2.812 & 3 & 1.781 \\
\hline Data34 & 100 & 261 & 4 & 5.718 & 5 & 3.218 & 3 & 1.844 \\
\hline Data35 & 100 & 271 & 5 & 3.156 & 4 & 2.125 & 3 & 1.547 \\
\hline Data36 & 100 & 451 & 5 & 4.032 & 4 & 2.484 & 3 & 2.031 \\
\hline Data37 & 100 & 742 & 5 & 4.438 & 5 & 3.906 & 3 & 2.266 \\
\hline Data38 & 100 & 922 & 4 & 4.938 & 4 & 3.406 & 3 & 2.531 \\
\hline Data39 & 150 & 481 & 5 & 11.609 & 4 & 7.841 & 3 & 7.11 \\
\hline Data40 & 150 & 473 & 5 & 10.531 & 5 & 9.062 & 3 & 5.5 \\
\hline Data41 & 150 & 402 & 4 & 12 & 5 & 9.297 & 4 & 5.986 \\
\hline Data42 & 100 & 334 & 4 & 2.875 & 6 & 2.782 & 4 & 1.47 \\
\hline Data43 & 150 & 453 & 5 & 19.687 & 5 & 8.653 & 4 & 6.656 \\
\hline Data44 & 150 & 1064 & 5 & 16.204 & 4 & 14.468 & 4 & 7.86 \\
\hline Data45 & 200 & 514 & 5 & 21.828 & 4 & 18.672 & 4 & 10.797 \\
\hline Data46 & 200 & 654 & 5 & 21.469 & 6 & 16.016 & 4 & 11.516 \\
\hline Data47 & 200 & 644 & 5 & 24.047 & 5 & 15.972 & 4 & 12.422 \\
\hline Data48 & 200 & 664 & 5 & 22.89 & 8 & 39.281 & 4 & 11.437 \\
\hline Data49 & 200 & 519 & 5 & 43.188 & 4 & 18.266 & 4 & 13.67 \\
\hline Data50 & 200 & 701 & 4 & 26.188 & 5 & 18.375 & 4 & 15.61 \\
\hline
\end{tabular}

Algorithm 1: AB-MDST without local search and without degree constraint Algorithm 2: AB-MDST with local search but without degree constraint Algorithm 3: AB-MDST with local search and with degree constraint

\section{CONCLUSION}

In this paper we discussed an Ant-Based algorithm- AB-MDST without local search and without degree constraint (algorithm 1) to find minimum spanning degree spanning trees from different input graphs and gave two improved versions of the algorithm named AB-MDST with local search but without degree constraint (algorithm 2) and AB-MDST with local search and with degree constraint (algorithm 3). The experimental results show that for both parameters (Degree and Time); algorithm 3 gives much better result than the other two algorithms.

\section{REFERENCES}

[1]. Dorigo, M., V. Maniezzo, and A. Colorni, "Ant System: Optimization by a Colony of Cooperating Agents," IEEE Trans. on Systems, Man, and Cybernetics - Part B, 26(1), Feb. 1996, pp. 29-41.

[2]. Volgenant, A., “A Lagrangean Approach to the Degree-Constrained Minimum Spanning Tree Problem," European Journal of Operational Research, 39, 1989, pp. 325-331.

[3]. Eila Safari and Azizallah Rahmati, "Using Learning Automata to Solving Degree-constrained Minimum Spanning Tree Problem", Australian Journal of Basic and Applied Sciences, 5(6): 337-341, 2011, ISSN 1991-8178

[4]. Minh N. Doan," An Effective Ant-Based Algorithm for the Degree-Constrained Minimum Spanning Tree Problem”, 1-4244-13400/07\$25.00_c 2007 IEEE

[5]. YOON-TECK BAU, CHIN-KUAN HO AND HONG-TAT EWE, "Ant Colony Optimization Approaches to the Degreeconstrained Minimum Spanning Tree Problem”, JOURNAL OF INFORMATION SCIENCE AND ENGINEERING 24, 1081 -1094 (2008)

[6]. Thang N. Bui and Catherine M. Zrncic, "An Ant-Based Algorithm for Finding Degree-Constrained Minimum Spanning Tree", GECCO’06, July 8-12, 2006, Seattle, Washington, USA.

[7]. Kamalika Chaudhuri, Satish Rao, Samantha Riesenfeld, and Kunal Talwar, "A Push-Relabel Algorithm for Approximating Degree Bounded MSTs"

[8]. Savelsbergh, M. and T. Volgenant, "Edge Exchanges in the Degree-Constrained Minimum Spanning Tree Problem," Computers and Operations Research, 12(4), 1985, pp. 341-348.

[9]. Marck De Berg, Marc Van Kreveld and Mark Overmars, "Computational Geometry Algorithms and Applications".

[10]. Marco Dorigo, Mauro Birattari, and Thomas Stutzle, "Ant Colony Optimization-Artificial Ants as a Computational Intelligence Technique". 